Supporting Information

\title{
Molecular Catalysis of Electrochemical Reactions. Overpotential and Turnover Frequency: Unidirectional and Bidirectional Systems.
}

\author{
Cyrille Costentin ${ }^{a, b}$ \\ ${ }^{a}$ Département de Chimie Moléculaire, Université Grenoble-Alpes, CNRS, UMR 5250, 38000 \\ Grenoble, France. ${ }^{b}$ Université de Paris, 75013 Paris, France.
}

cyrille.costentin@univ-grenoble-alpes.fr 


\section{One electron-one step bidirectional (reversible) system:}

We consider the reaction scheme depicted in scheme S1.

Scheme S1. One electron-one step mechanism

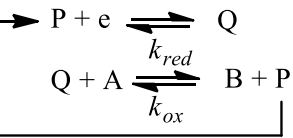

From the derivation in reference $1 \mathrm{~S}$, the $\mathrm{CV}$ equation in canonical conditions is:

$$
\begin{gathered}
\frac{i}{F}=\left\{\frac{k_{r e d} C_{\mathrm{A}}^{0}}{1+\exp \left[\frac{F\left(E-E_{\mathrm{cat}}^{0}\right)}{R T}\right]}-\frac{k_{o x} C_{\mathrm{B}}^{0}}{1+\exp \left[-\frac{F\left(E-E_{\mathrm{cat}}^{0}\right)}{R T}\right]}\right\} C_{\mathrm{cat}}^{0} \times S \times \mu_{r e v} \\
\text { with } \\
\mu_{r e v}=\sqrt{\frac{D_{\text {cat }}}{k_{\text {red }} C_{\mathrm{A}}^{0}+k_{o x} C_{\mathrm{B}}^{0}}}
\end{gathered}
$$

\section{Concentration profiles:}

According to the Nernst law, concentrations at the electrode surfaces are:

$$
\left(C_{\mathrm{Q}}\right)_{0}=\frac{C_{\text {cat }}^{0}}{1+\exp \left[\frac{F\left(E-E_{\text {cat }}^{0}\right)}{R T}\right]} \text { and }\left(C_{\mathrm{P}}\right)_{0}=\frac{C_{\text {cat }}^{0}}{1+\exp \left[-\frac{F\left(E-E_{\text {cat }}^{0}\right)}{R T}\right]}
$$

Equilibrium $\mathrm{A}+\mathrm{Q}=\mathrm{B}+\mathrm{P}$ in the solution bulk leads to the following concentrations for $\mathrm{P}$ and $\mathrm{Q}$ in the solution:

$$
C_{\mathrm{Q}}^{e q}=\frac{C_{\mathrm{cat}}^{0}}{1+\exp \left[\frac{F\left(E_{\text {target }}^{0}+\frac{R T}{F} \ln \frac{C_{\mathrm{A}}^{0}}{C_{\mathrm{B}}^{0}}-E_{\text {cat }}^{0}\right)}{R T}\right]} \text { and } C_{\mathrm{P}}^{e q}=\frac{C_{\text {cat }}^{0}}{1+\exp \left[-\frac{F\left(E_{\text {target }}^{0}+\frac{R T}{F} \ln \frac{C_{\mathrm{A}}^{0}}{\left.C_{\mathrm{B}}^{0}-E_{\text {cat }}^{0}\right)}\right.}{R T}\right]}
$$

We note that $\eta_{\text {eff }}=E_{\text {cat }}^{0}-E_{\text {target }}^{e q}$ and $E_{\text {target }}^{e q}=E_{\text {target }}^{0}+\frac{R T}{F} \ln \frac{C_{\mathrm{A}}^{0}}{C_{\mathrm{B}}^{0}}$

From the derivation in reference $1 \mathrm{~S}$, we have:

$C_{\mathrm{Q}}=\left(\left(C_{\mathrm{Q}}\right)_{0}-C_{\mathrm{Q}}^{e q}\right) \exp \left\{-x \sqrt{\frac{k_{r e d} C_{\mathrm{A}}^{0}}{D_{\text {cat }}}} \sqrt{1+\exp \left[\frac{F}{R T}\left(E_{\text {cat }}^{0}-E_{\text {target }}^{0}-\frac{R T}{F} \ln \frac{C_{\mathrm{A}}^{0}}{C_{\mathrm{B}}^{0}}\right)\right]}\right\}+C_{\mathrm{Q}}^{e q}$

Noting that $\frac{k_{o x} C_{\mathrm{B}}^{0}}{k_{\text {red }} C_{\mathrm{A}}^{0}}=\exp \left[\frac{F}{R T}\left(E_{\text {cat }}^{0}-E_{\text {target }}^{0}-\frac{R T}{F} \ln \frac{C_{\mathrm{A}}^{0}}{C_{\mathrm{B}}^{0}}\right)\right]$, we obtain:

$C_{\mathrm{Q}}(x)=\left[\left(C_{\mathrm{Q}}\right)_{0}-C_{\mathrm{Q}}^{e q}\right] \exp \left(-\frac{x}{\mu_{\text {red }}}\right)+C_{\mathrm{Q}}^{e q}$ and $C_{\mathrm{P}}(x)=C_{\text {cat }}^{0}-C_{\mathrm{Q}}(x)$.

Turnover frequency: 
As indicated in the text, $T O F_{\text {red }}$ (respectively $T O F_{o x}$ ) is defined as the number of moles of product B (respectively A) generated per unit of time by the cathodic (respectively anodic) current, i.e. $i_{\text {cathodic }} / F$ (respectively $\left|i_{\text {anodic }}\right| / F$ ) divided by the maximal number of moles of active catalyst formed by the polarization of the electrode.

$T O F_{\text {red }}=\frac{i_{\text {cathodic }} / F}{N_{\max }}$

with $N_{\max }=S \int_{0}^{\infty}\left[\left(C_{\mathrm{Q}, p l}(x)-C_{\mathrm{Q}}^{e q}\right)+\left(C_{\mathrm{P}}^{e q}-C_{\mathrm{P}, p l}(x)\right) \frac{k_{o x} C_{\mathrm{B}}^{0}}{k_{r e d} C_{\mathrm{A}}^{0}}\right] d x$

From the above concentration profiles expressions:

$$
\begin{aligned}
& C_{\mathrm{Q}, \mathrm{pl}}(x)-C_{\mathrm{Q}}^{e q}=\left[C_{\text {cat }}^{0}-C_{\mathrm{Q}}^{e q}\right] \exp \left(-\frac{x}{\mu_{\text {red }}}\right) \text { and } C_{\mathrm{P}}^{e q}-C_{\mathrm{P}, p l}(x)=C_{\mathrm{P}}^{e q} \exp \left(-\frac{x}{\mu_{\text {red }}}\right)=\left[C_{\text {cat }}^{0}-C_{\mathrm{Q}}^{e q}\right] \exp \left(-\frac{x}{\mu_{\text {red }}}\right) \text {, hence: } \\
& N_{\max }=S \int_{0}^{\infty}\left[\left[C_{\mathrm{cat}}^{0}-C_{\mathrm{Q}}^{e q}\right] \exp \left(-\frac{x}{\mu_{\text {red }}}\right)+\left[C_{\mathrm{cat}}^{0}-C_{\mathrm{Q}}^{e q}\right] \exp \left(-\frac{x}{\mu_{\text {red }}}\right) \frac{k_{o x} C_{\mathrm{B}}^{0}}{k_{r e d} C_{\mathrm{A}}^{0}}\right] d x, \text { leading to: } \\
& N_{\max }=S\left(\frac{k_{r e d} C_{\mathrm{A}}^{0}+k_{o x} C_{\mathrm{B}}^{0}}{k_{\text {red }} C_{\mathrm{A}}^{0}}\right)\left(C_{\text {cat }}^{0}-C_{\mathrm{Q}}^{e q}\right) \mu_{\text {red }}=S \times \mu_{\text {red }} \times C_{\text {cat }}^{0} \text { taking into account: } \\
& C_{\mathrm{Q}}^{e q}=\frac{C_{\mathrm{cat}}^{0}}{1+\exp \left[\frac{F\left(E_{\mathrm{target}}^{0}+\frac{R T}{F} \ln \frac{C_{\mathrm{A}}^{0}}{\left.C_{\mathrm{B}}^{0}-E_{\mathrm{cat}}^{0}\right)}\right.}{R T}\right]}=\frac{k_{o x} C_{\mathrm{B}}^{0}}{k_{r e d} C_{\mathrm{A}}^{0}+k_{o x} C_{\mathrm{B}}^{0}} C_{\mathrm{cat}}^{0}
\end{aligned}
$$

Thus, recalling that $\frac{i_{\text {cathodic }}}{F S}=\frac{k_{\text {red }} C_{\mathrm{A}}^{0}}{1+\exp \left[\frac{F\left(E-E_{\text {cat }}^{0}\right)}{R T}\right]} C_{\text {cat }}^{0} \times \mu_{\text {rev }}$, we obtain:

$$
T O F_{\text {red }}=\frac{k_{\text {red }} C_{\mathrm{A}}^{0}}{1+\exp \left[\frac{F\left(E-E_{\text {cat }}^{0}\right)}{R T}\right]}
$$




\section{Two electron-two step bidirectional $\mathrm{EC}_{\mathrm{r}} \mathrm{EC}_{\mathrm{r}}$ system:}

We consider the reaction scheme depicted in scheme $\mathrm{S} 2$.

Scheme S2. Bidirectional two electron-two step mechanism

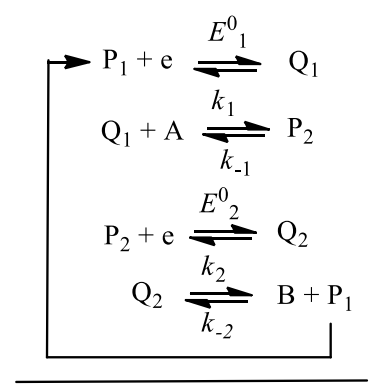

$$
\mathrm{A}+2 \mathrm{e} \rightleftharpoons \mathrm{B}
$$

Considering an excess of both $\mathrm{A}$ and $\mathrm{B}$ in solution, the $\mathrm{CV}$ may be obtained by resolution of the following set of partial derivative equations.

$$
\begin{aligned}
& \frac{\partial\left[\mathrm{P}_{1}\right]}{\partial t}=D_{c a t} \frac{\partial^{2}\left[\mathrm{P}_{1}\right]}{\partial x^{2}}-k_{-2} C_{\mathrm{B}}^{0}\left[\mathrm{P}_{1}\right]+k_{2}\left[\mathrm{Q}_{2}\right] \\
& \frac{\partial\left[\mathrm{Q}_{1}\right]}{\partial t}=D_{c a t} \frac{\partial^{2}\left[\mathrm{Q}_{1}\right]}{\partial x^{2}}-k_{1} C_{\mathrm{A}}^{0}\left[\mathrm{Q}_{1}\right]+k_{-1}\left[\mathrm{P}_{2}\right] \\
& \frac{\partial\left[\mathrm{P}_{2}\right]}{\partial t}=D_{c a t} \frac{\partial^{2}\left[\mathrm{P}_{2}\right]}{\partial x^{2}}-k_{-1}\left[\mathrm{P}_{2}\right]+k_{1} C_{\mathrm{A}}^{0}\left[\mathrm{Q}_{1}\right] \\
& \frac{\partial\left[\mathrm{Q}_{2}\right]}{\partial t}=D_{c a t} \frac{\partial^{2}\left[\mathrm{Q}_{2}\right]}{\partial x^{2}}-k_{2}\left[\mathrm{Q}_{2}\right]+k_{-2} C_{\mathrm{B}}^{0}\left[\mathrm{P}_{1}\right]
\end{aligned}
$$

with, as initial and boundary conditions:

$t=0, x \geq 0$ and $x=\infty, x \geq 0$ :

$\left[\mathrm{P}_{1}\right]=C_{\mathrm{P}_{1}}^{e q},\left[\mathrm{Q}_{1}\right]=C_{\mathrm{Q}_{1}}^{e q},\left[\mathrm{P}_{2}\right]=C_{\mathrm{P}_{2}}^{e q},\left[\mathrm{Q}_{2}\right]=C_{\mathrm{Q}_{2}}^{e q}$, corresponding to the solution equilibria:

$k_{-2} C_{\mathrm{B}}^{0} C_{\mathrm{P}_{1}}^{e q}=k_{2} C_{\mathrm{Q}_{2}}^{e q}$

$k_{1} C_{\mathrm{A}}^{0} C_{\mathrm{Q}_{1}}^{e q}=k_{-1} C_{\mathrm{P}_{2}}^{e q}$

and $C_{\mathrm{P}_{1}}^{e q}+C_{\mathrm{Q}_{2}}^{e q}+C_{\mathrm{Q}_{1}}^{e q}+C_{\mathrm{P}_{2}}^{e q}=C_{\text {cat }}^{0}$

$x=0, t \geq 0: \frac{i_{1}}{F S D_{\text {cat }}}=\left(\frac{\partial\left[\mathrm{P}_{1}\right]}{\partial x}\right)_{x=0}=-\left(\frac{\partial\left[\mathrm{Q}_{1}\right]}{\partial x}\right)_{x=0}, \frac{i_{2}}{F S D_{\text {cat }}}=\left(\frac{\partial\left[\mathrm{P}_{2}\right]}{\partial x}\right)_{x=0}=-\left(\frac{\partial\left[\mathrm{Q}_{1}\right]}{\partial x}\right)_{x=0}, i=i_{1}+i_{2}$

$\frac{\left[\mathrm{P}_{1}\right]_{x=0}}{\left[\mathrm{Q}_{1}\right]_{x=0}}=\exp \left[\frac{F}{R T}\left(E-E_{1}^{0}\right)\right], \frac{\left[\mathrm{P}_{2}\right]_{x=0}}{\left[\mathrm{Q}_{2}\right]_{x=0}}=\exp \left[\frac{F}{R T}\left(E-E_{2}^{0}\right)\right]$

Resolution of the problem can be obtained by considering Laplace transform and the dimensionless parameters:

$y=\frac{x}{\sqrt{D_{c a t} R T / F v}} ; p_{i}=\frac{\left[\mathrm{P}_{1}\right]}{C_{\text {cat }}^{0}} ; q_{i}=\frac{\left[\mathrm{Q}_{1}\right]}{C_{\text {cat }}^{0}} ; s$ is the variable is the Laplace space. 


$$
\begin{aligned}
& \left(\frac{k_{-2} C_{\mathrm{B}}^{0}}{F v / R T}+s\right) \bar{p}_{1}-\frac{k_{2}}{F v / R T} \bar{q}_{2}-\frac{C_{\mathrm{P}_{1}}^{e q}}{C_{\text {cat }}^{0}}=\frac{\partial^{2} \bar{p}_{1}}{\partial y^{2}} \\
& \left(\frac{k_{2}}{F v / R T}+s\right) \bar{q}_{2}-\frac{k_{-2} C_{\mathrm{B}}^{0}}{F v / R T} \bar{p}_{1}-\frac{C_{\mathrm{Q}_{2}}^{e q}}{C_{\text {cat }}^{0}}=\frac{\partial^{2} \bar{q}_{2}}{\partial y^{2}}
\end{aligned}
$$

and

$$
\begin{aligned}
& \left(\frac{k_{1} C_{\mathrm{A}}^{0}}{F v / R T}+s\right) \bar{q}_{1}-\frac{k_{-1}}{F v / R T} \bar{p}_{2}-\frac{C_{\mathrm{Q}_{1}}^{e q}}{C_{\text {cat }}^{0}}=\frac{\partial^{2} \bar{q}_{1}}{\partial y^{2}} \\
& \left(\frac{k_{-1}}{F v / R T}+s\right) \bar{p}_{2}-\frac{k_{1} C_{\mathrm{A}}^{0}}{F v / R T} \bar{q}_{1}-\frac{C_{\mathrm{P}_{2}}^{e q}}{C_{\text {cat }}^{0}}=\frac{\partial^{2} \bar{p}_{2}}{\partial y^{2}}
\end{aligned}
$$

Pure kinetics conditions entails to consider $\frac{k_{-2} C_{\mathrm{B}}^{0}}{F v / R T}>s ; \frac{k_{2}}{F v / R T}>s ; \frac{k_{1} C_{\mathrm{A}}^{0}}{F v / R T}>>s ; \frac{k_{-1}}{F v / R T} \gg s$. Therefore, going back to the real space, we have to solve the steady-state system:

$$
\begin{aligned}
& D_{c a t} \frac{d^{2}\left[\mathrm{P}_{1}\right]}{\partial x^{2}}=k_{-2} C_{\mathrm{B}}^{0}\left[\mathrm{P}_{1}\right]-k_{2}\left[\mathrm{Q}_{2}\right] \\
& D_{c a t} \frac{d^{2}\left[\mathrm{Q}_{2}\right]}{\partial x^{2}}=k_{2}\left[\mathrm{Q}_{2}\right]-k_{-2} C_{\mathrm{B}}^{0}\left[\mathrm{P}_{1}\right]
\end{aligned}
$$

and

$$
\begin{aligned}
& D_{c a t} \frac{d^{2}\left[\mathrm{Q}_{1}\right]}{\partial x^{2}}=k_{1} C_{\mathrm{A}}^{0}\left[\mathrm{Q}_{1}\right]-k_{-1}\left[\mathrm{P}_{2}\right] \\
& D_{c a t} \frac{d^{2}\left[\mathrm{P}_{2}\right]}{\partial x^{2}}=k_{-1}\left[\mathrm{P}_{2}\right]-k_{1} C_{\mathrm{A}}^{0}\left[\mathrm{Q}_{1}\right]
\end{aligned}
$$

Solving the above coupled systems taking into account boundary conditions and noting that at steady-state $i_{1}=i_{2}=i / 2$, we obtain at the cathodic plateau ( $E \rightarrow-\infty$ ) the following concentration profiles:

$$
\begin{aligned}
& C_{\mathrm{P}_{1}, p l}=C_{\mathrm{P}_{1}}^{e q}-\frac{i_{\text {pl,cathodic }}}{2 F S \sqrt{D_{\text {cat }}}} \frac{1}{\sqrt{k_{2}+k_{-2} C_{\mathrm{B}}^{0}}} \exp \left(-x \sqrt{\frac{k_{2}+k_{-2} C_{\mathrm{B}}^{0}}{D_{c a t}}}\right) \\
& C_{\mathrm{Q}_{1}, p l}=C_{\mathrm{Q}_{1}}^{e q}+\frac{i_{\text {pl,cathodic }}}{2 F S \sqrt{D_{\text {cat }}}} \frac{1}{\sqrt{k_{1} C_{\mathrm{A}}^{0}+k_{-1}}} \exp \left(-x \sqrt{\frac{k_{1} C_{\mathrm{A}}^{0}+k_{-1}}{D_{c a t}}}\right) \\
& C_{\mathrm{P}_{2}, p l}=C_{\mathrm{P}_{2}}^{e q}-\frac{i_{\text {pl,cathodic }}}{2 F S \sqrt{D_{\text {cat }}}} \frac{1}{\sqrt{k_{1} C_{\mathrm{A}}^{0}+k_{-1}}} \exp \left(-x \sqrt{\frac{k_{1} C_{\mathrm{A}}^{0}+k_{-1}}{D_{c a t}}}\right) \\
& C_{\mathrm{Q}_{2}, p l}=C_{\mathrm{Q}_{2}}^{e q}+\frac{i_{\text {pl,cathodic }}}{2 F S \sqrt{D_{\text {cat }}}} \frac{1}{\sqrt{k_{2}+k_{-2} C_{\mathrm{B}}^{0}}} \exp \left(-x \sqrt{\frac{k_{2}+k_{-2} C_{\mathrm{B}}^{0}}{D_{c a t}}}\right)
\end{aligned}
$$

Transposition to the anodic plateau $(E \rightarrow \infty)$ is straightforward.

\section{Maximal turnover frequency:}


As indicated in the text, $T O F_{r e d}^{\max }$ (respectively $T O F_{o x}^{\max }$ ) is defined as the number of moles of product B (respectively A) generated per unit of time by the maximal cathodic (respectively anodic) current, i.e. $i_{\text {pl,cathodic }} / 2 F$ (respectively $\left|i_{\text {anodic }}\right| / 2 F$ ) divided by the maximal number of moles of active catalyst formed by the polarization of the electrode.

TOF $_{\text {red }}^{\max }=\frac{i_{\text {pl,cathodic }} / 2 F}{N_{1, \max }+N_{2}, \max }$

with

$$
N_{1}, \max +N_{2}, \max =S \int_{0}^{\infty}\left[\left(C_{\mathrm{Q}_{1}, p l}-C_{\mathrm{Q}_{1}}^{e q}\right)+\frac{k_{-1}}{k_{1} C_{\mathrm{A}}^{0}}\left(C_{\mathrm{P}_{2}}^{e q}-C_{\mathrm{P}_{2}, p l}\right)\right] d x+S \int_{0}^{\infty}\left[\left(C_{\mathrm{Q}_{2}, p l}-C_{\mathrm{Q}_{2}}^{e q}\right)+\frac{k_{-2} C_{\mathrm{B}}^{0}}{k_{2}}\left(C_{\mathrm{P}_{1}}^{e q}-C_{\mathrm{P}_{1}, p l}\right)\right] d x
$$

Considering the concentration profiles obtained above, we have:

$$
\begin{gathered}
C_{\mathrm{Q}_{2}, p l}-C_{\mathrm{Q}_{2}}^{e q}=C_{\mathrm{P}_{1}}^{e q}-C_{\mathrm{P}_{1}, p l}=\frac{i_{\text {pl,cathodic }}}{2 F S \sqrt{D_{\text {cat }}}} \frac{1}{\sqrt{k_{2}+k_{-2} C_{\mathrm{B}}^{0}}} \exp \left(-x \sqrt{\frac{k_{2}+k_{-2} C_{\mathrm{B}}^{0}}{D_{c a t}}}\right) \\
C_{\mathrm{Q}_{1}, p l}-C_{\mathrm{Q}_{1}}^{e q}=C_{\mathrm{P}_{2}}^{e q}-C_{\mathrm{P}_{2}, p l}=\frac{i_{\text {pl,cathodic }}}{2 F S \sqrt{D_{\text {cat }}}} \frac{1}{\sqrt{k_{1} C_{\mathrm{A}}^{0}+k_{-1}}} \exp \left(-x \sqrt{\frac{k_{1} C_{\mathrm{A}}^{0}+k_{-1}}{D_{c a t}}}\right)
\end{gathered}
$$

Thus:

$$
N_{1}, \max +N_{2}, \max =\left(\frac{1}{k_{1} C_{\mathrm{A}}^{0}}+\frac{1}{k_{2}}\right) \frac{i_{\text {pl,cathodic }}}{2 F}
$$

Finally leading to:

$$
T O F_{\text {red }}^{\max }=\frac{k_{1} C_{\mathrm{A}}^{0} \times k_{2}}{k_{1} C_{\mathrm{A}}^{0}+k_{2}}
$$

Similarly, we have $T O F_{o x}^{\max }=\frac{k_{-2} C_{\mathrm{B}}^{0} \times k_{-1}}{k_{-2} C_{\mathrm{B}}^{0}+k_{-1}}$ 


\section{References:}

(1S) Savéant, J-M. Molecular Catalysis of Electrochemical Reactions. Cyclic Voltammetry of Systems Approaching Reversibility. ACS Catal. 2018, 8, 7608-7611. 